\title{
M. Pusterla*
}

October 13, 1981

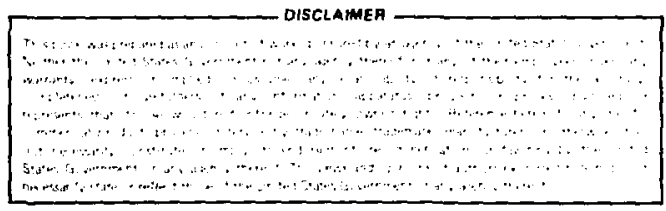

"Parmanant address: Istituta Fisica Universita' di Padova, 35100 Padova [ibly].

\author{
ACCELERATOR DEPARTMENT \\ BROOKHAVEN NATIONAL LABORATORY \\ UPTON, NEW YORK 11973
}




\begin{abstract}
A proposal of using extrapolation algorithms as an analytical numerical tool for the evaluation of the beam-beam effects in storage rings is formulated. These algorithms already known to experts of Applied Mathematics, are described in some detail.

Physical models for the beam-beam interaction effects on betatron motion and polarization of a test particle are discussed and put in such a form to allow the application of the extrapolation algorithms.

ACKNOWLEDGMENTS
\end{abstract}

The author wishes to thank Dr. E. Courant for stimulating discussions on the subject. He acknowledges the interest and collaboration of Drs. M. Cornacchia and A. Luccio, and thanks Dr. J.C. Herrera for valuable discussions on several topics concerning the ISABELlE case. He would al so like to thank J. Ferrero and D. Votruba for their excellent typing. 


\section{INTRODUCTION}

The detailed knowledge of the temporal evolution of a given system that is ruled by deterministic dynamical laws, in a certain finite interval of time, contains all the information necessary to predict its future behavior and, in particular, at asymptotic time values. However, the time functional dependence of the evolution is explicitly computable analytically in a very limited number of physical systems: most realistic cases have evolution functions that are known only numerically and involve a certain amount of indetermination.

In such a situation the extrapolation to asymptotic time values becomes delicate and requires particular methods of numerical analysis that are able to avoid, as much as possible, the dispersion of the initial information, thus providing an extrapolated temporal prediction that is meaningful and keeps a deterministic relation with the initial input.

It is fortunate that, nowadays, applied mathematics offers various algoritims which can do a limit extrapolation of this type. The fact that many algorithms are available, based on different mathematical principles, leads one to invest their predictions with a great degree of confidence whenever they converge independent ly toward a unique limit. Approaches of this type are particularly interesting in stability problems: one has to induce the regime conditions (or asymptotic conditions) of a system from a large but finite set of data that represent all the information obtained from experimental measurements or simulation computations.

This report is concerned with stability problems in classical mechanics, especially as they apply to the beam-bean interaction effects on the stability of beams in storage rings (ISABELLE is a particular case), but the method illustrated is general and, in our opinion, can be used to search for the stability conditions in various cases.

First, the extrapolation algorithms that are available at present are explained. A subsequent section deals with their application to the beamis in storage rings (with particular attention to ISABELLE).

\section{EXTRAPOLATION ALGORITHMS}

If we are given a sequence $\left(S_{n}\right)$, cften depending on a sequence of parameters $\left(X_{n}\right)$, the relevant point consists of knowing whether it converges or not, and, in case of convergence, the determination of its limit $S$. 
Under the hypothesis of convergence we define the error $S_{n}-S$ in the following form:

$$
s_{n}-s=a_{a} g_{1}(n)+a_{2} g_{2}(n)+\cdots
$$

where the $g_{i}(n)$ are given sequences; accurate approximations to $S$ are obtained by using extrapolation methods.

We here illustrate a general procedure that is able to provide us with several extrapolation methods: this formalism includes most of the sequence transformations actually used for the acceleration of the convergence. Although we deal, for simplicity, with scalar sequences $\left(s_{n}\right)$, the generalization to m-dimensional vectors and matrices is fairly straight forward.

Linear extrapolation processes. Let us assume that the sequence $\left(s_{n}\right)$ behaves in the following way:

$$
s_{n}=s+a_{1} g_{1}(n)+a_{2} g_{2}(n)+\ldots+a_{k} g_{k}(n)
$$

where the $g_{i}$ are given; in general $S$ is an approximate value of the limit of $\left(S_{n}\right)$ if it converges, or an approximate value of its anti-limit if it diverges. The calculation of $S$ follows immediately from the solution of the linear system (which we consider for simplicity non-singular):

$$
\begin{gathered}
s_{n+i}=s+a_{1} g_{1}(n+i)+\ldots+a_{k} g_{k}(n+i) \\
i=0,1, \ldots, k .
\end{gathered}
$$

If the sequence $\left(s_{n}\right)$ does not verify Eq. (2) exactly, then the value of $S$ derived from the preceding linear systent depends on the indices $m$ and $k$. Let us call it $E_{k}\left(s_{n}\right)$. We have, from $E q .(3)$,

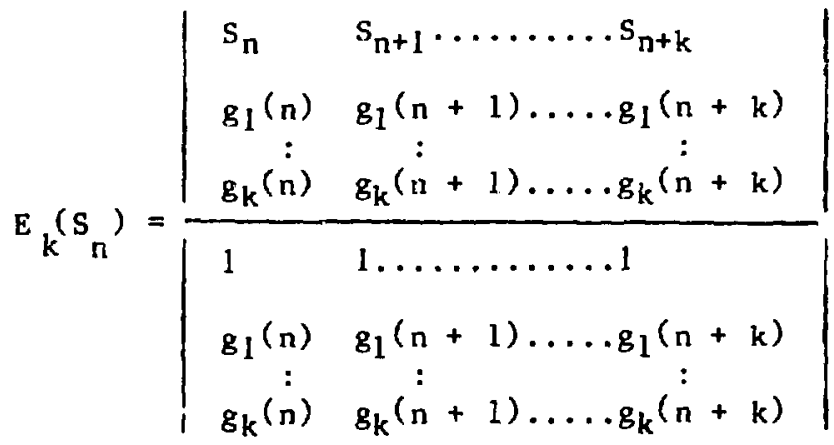

Various choices of the functions $g_{i}(n)$ allow us to reproduce well-known transformations which may be used to accelerate the convergence of the original sequence $\left(s_{n}\right)$. 
The formulation includes also a very important subset of interpolations, discussed later in detail, namely the rational interpolations. Let us take the fraction

$$
s_{n}=\frac{s+b_{1} f_{1}(n)+b_{2} f_{2}(n)+\ldots+b_{m m} f_{m}(n)}{1+c_{1} h_{1}(n)+c_{2} h_{2}(n)+\ldots+c_{p} h_{p}(n)} .
$$

We may reduce this formula to Eq. (2) by setting $g_{1}(n)=f_{1}(n)$, $g_{2}(n)=f_{2}(n), \ldots, g_{m}(n)=f_{m}(n) ; g_{m+1}(n)=s_{n} h_{1}(n)$, $g_{m+2}(n)=s_{n} h_{2}(n), \ldots, g_{m+p}(n)=s_{n} h_{p}(n)$. A particular case of rational interpolation-extrapolation is represented by rational fractions which have their numerators and denominators as simple polynomials of $x_{n}$ : $f_{i}(n)=h_{j}(n)=x_{n}^{-i}$ for $i=1,2, \ldots, k / 2$.

Wherever the sequences $g_{i}$ do not depend on $\left(s_{n}\right)$ we deal with a linear sequence transformation, whereas the transformation is nonlinear if the $g_{i}$ 's depend on $\left(s_{n}\right)$.

The E-Algorithm. ${ }^{1}$ The computation of the numbers $E_{k}\left(S_{n}\right)$ for all $k$ and $n$ can be accomplished by the following recursive algorithm (E-algorithm):

$$
\begin{array}{ll}
E_{0}^{(n)}=s_{n} & n=0,1, \ldots \\
g_{0, i}^{(n)}=g_{i}(n) & i=1,2, \ldots \quad n=0,1, \ldots
\end{array}
$$

and for $k=1,2, \ldots ; n=0,1, \ldots$.

$$
\begin{gathered}
F_{k}^{(n)}=\frac{E_{k-1}^{(n)} g_{k-1, k}^{(n+1)}-E_{k-1}^{(n+1)} g_{k-1, k}^{(n)}}{g_{k-1, k}^{(n+1)}-g_{k-1, k}^{(n)}}, \\
g_{k, i}^{(n)}=\frac{g_{1,-1, j}^{(n)} g_{k-1, k}^{(n+1)}-g_{k-1, i}^{(n+1)} g_{k-1, k}^{(n)}}{g_{k-1, k}^{(n+1)}-g_{k-1, k}^{(n)}}, i=k+1, k+2, \ldots(7)
\end{gathered}
$$

where $E_{k}^{(n)}$ coincides identically with $E_{k}\left(S_{n}\right)$.

The fundamental algebraic property of the general extrapolation defined above is summarized by the following theorem: if

$$
s_{n}=s+a_{l} g_{l}(n)+\ldots+a_{k} g_{k}(n), \forall_{n}
$$

then

$$
E_{k}\left(s_{n}\right)=s, \forall_{n}
$$


and, more generally,

if

$$
s_{n}=s+a_{1} g_{l}(n)+\ldots+a_{k} g_{k}(n)+\ldots y_{n}
$$

then

$$
E_{k}\left(s_{n}\right)=s+a_{k+1} g_{k, k+1}^{(n)}+a_{k+2} g_{k, k+2}^{(n)}+\ldots, v_{k, n} .
$$

Convergence theorems. ${ }^{1}$ Convergence of the $E_{k}^{(n)}$ sequence in the various columns at fixed $k$ and its degree of acceleration in comparison with the initial sequence $\left(s_{n}\right)$ are characterized by a set of theorems.

Theorem 1. If $\lim _{n \rightarrow \infty} E_{k-1}\left(s_{n}\right)=s$ and if $\alpha$ and $\beta$ exist such that $0<\alpha<1<\beta$ and $\left.\lg _{k-1, k}^{(n+1)} / g_{k-1, k}^{(n)}\right] \notin(\alpha, \beta) \forall_{n}>N$, then $\lim _{n \rightarrow \infty} E_{k}\left(s_{n}\right)=s$.

Theorem 2. If $\lim _{n \rightarrow \infty} S_{n}=S$, if $\lim _{n \rightarrow \infty}\left[g_{i}(n+1) / g_{i}(n)\right]=b_{i} \neq 1$, and if $b_{i} \neq b_{j} \neq i \neq j$, the $\lim _{n \rightarrow \infty} E_{k}\left(S_{n}\right)=s \neq k$.

Theorem 3. Under the conditions of theorem 2, if $\lim _{n \rightarrow \infty}\left[E_{k-1}\left(s_{n+1}\right)-s\right] /\left[E_{k-1}\left(s_{n}\right)-s\right]=b_{k}$, then $E_{k}\left(s_{n}\right)-s$ $=0\left[E_{k-1}\left(S_{n}\right)-S\right]$.

Theorem 4. If the conditions of theorem 2 are satisfied, if $g_{i+1}(n)=o\left[g_{i}(n)\right] \forall i$, and if $s_{n}=s+a_{1} g_{1}(n)$

$+a_{2} g_{2}(n)+\ldots$, then $E_{k}\left(s_{n}\right)-s=o\left[E_{k-1}\left(S_{n}\right)-S\right] \forall k$.

The importance of Theorem 4 is evident. A family of sequences satisfying the condition $g_{i+1}(n)=o\left[g_{i}(n)\right]$ is called a scale of comparison, and $a_{1} g_{1}(n)+a_{2} g_{2}(n)+\ldots$ is the asymptotic expansion of $S_{n}-S$ with respect to it. Theorem 4 guarantees that sequences whose error has an asymptotic expansion with respect to a given scale of comparison can be accelerated by an extrapolation process (condition sufficient but not necessary).

\section{RELEVANT CASES FOR APPLICATIONS}

From the E-algorithm, various extrapolation techniques are easily obtained which have been known for a long time and are still considered 
the standard methods to start from in order to search for the limit of a sequence. We here refer to the so-called $\varepsilon-, \rho^{-}$, and r-algorithms, and finally the $\theta$-algorithm.

-Algorithm. If we are given a sequence $s_{0}, s_{1}, \ldots$, we may relate its elements to the partial sums of a power series: we have the function

$$
f(\lambda)=s_{0}+\left(s_{1}-s_{0}\right) \lambda+\left(s_{2}-s_{1}\right) \lambda^{2}+\ldots
$$

and define

$$
f_{n}(\lambda)=s_{0}+\sum_{0}^{n-1} \Delta s_{i} \lambda^{i+1}
$$

where $s_{i}=s_{i+1}-s_{i}$; thus $f_{n}(1)=s_{n}$.

The Pade approximant $[k, k+j]$ of $f(\lambda)$, calculated at $\lambda=1$, defines the : $\frac{i}{2 k}$ sequence:

$$
\varepsilon_{2 k}^{i}=\left[k, k+\left.j(\lambda)\right|_{\lambda=1} .\right.
$$

From the standard Pade - representation we get:

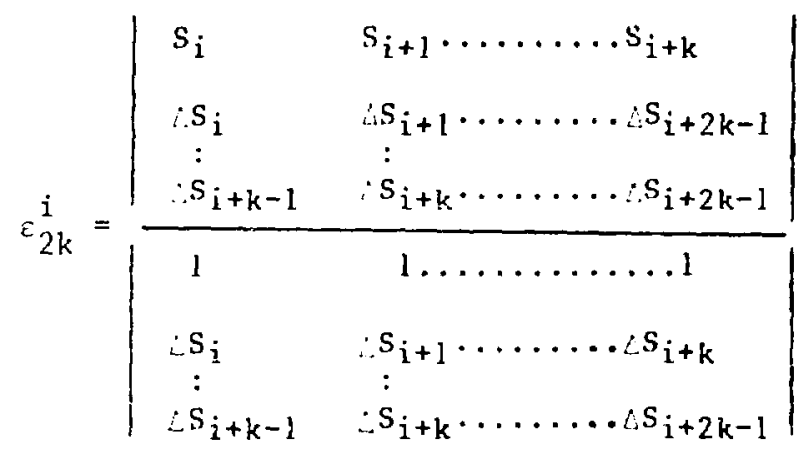

Equation (11) coincides with the Shanks transformation defined by Eq. ( 10$)$. For $k=1$ we obtain $\varepsilon_{2}=\left(s_{i+1} s_{i}-s_{i+1}^{2}\right) /\left(s_{i+2}-2 s_{i+1}+s_{i}\right)$,
which is the familiar litken $s^{2}$ acceleration technique.

Historically Shanks ${ }^{2}$ generalized the $i^{2}$ and established its connection with the Pade table. The recursive formulae of the s-algorithm, first given by Wynn ${ }^{3}$, are

$$
\varepsilon_{0}^{i}=S_{i} ; \varepsilon_{-1}^{i}=0 ; \varepsilon_{k+1}^{i}=\varepsilon_{k-1}^{i+1}+\frac{1}{\varepsilon_{k}^{i+1}-\varepsilon_{k}^{i}} .
$$

The sequences $\left(\varepsilon_{k}^{i}\right)$ are normally set out in a triangular table: 


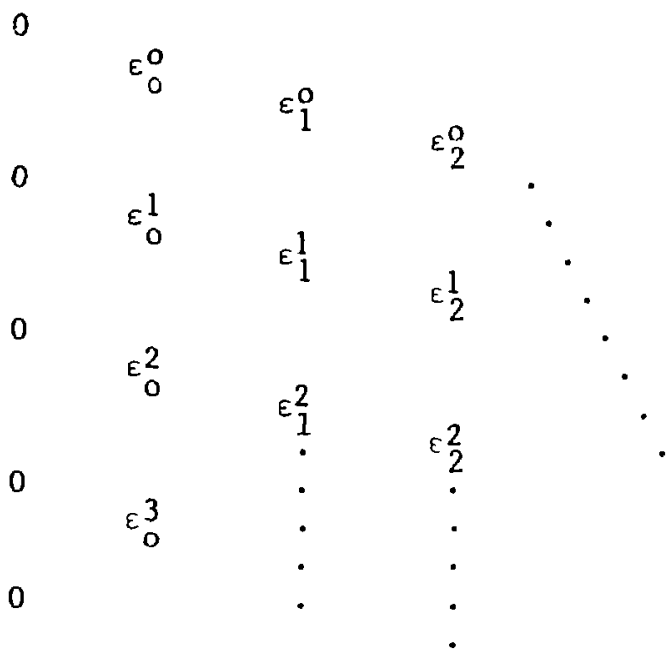

The columns that have even values of the low index, namely $\varepsilon_{2 k}^{i}$, are the ones that converge to the same limit as the initial sequence $\left(s_{i}\right)$ : they are strictly related with the Pade approximant method and use its convergence properties. The add index columns $\varepsilon_{2 k+1}^{1}$ represent an intermediate step of the calculation which it is necessary to compute in order to use the recursive procedure. If the initial sequence $\left(s_{i}\right)$ diverges, the algorithm may produce a convergent sequence from it and thus find an accumulation point which is not the limit; we call this the anti-limit.

Generalization to vector and matrix sequences. Any time one has to deal with vector or matrix sequences that tend to a vector or a matrix limit, one may use the $\varepsilon$-algorithm to accelerate them. In order to use it, we must first give a definition of vector inverse (and matrix inverse). A suitable choice is the Samuelson vector inverse: given an $n$-dimensional vector $\vec{x}\left(x_{1}, x_{2}, \ldots\right)$ we define the vector

$$
(\vec{x})^{-1}=\left(\vec{x} \cdot \vec{x}^{*}\right)^{-1} \cdot \vec{x}
$$

where $\vec{X}^{*}$ is the complex conjugate of $\vec{X}$. For matrices one can use either a similar definition or the usual matrix inverse (which appears computationally more expensive).

Applications of the $\varepsilon$-algorithm in the vector form are very general: let us consider the sequences $\left(\vec{x}_{i}\right)$ generated by some nonlinear functions $\vec{F}\left(\vec{X}_{i}\right)$ :

$$
\overrightarrow{\mathrm{X}}_{\mathrm{i}+1}=\overrightarrow{\mathrm{F}}\left(\overrightarrow{\mathrm{x}}_{\mathbf{i}}\right) \text {. }
$$

It has been proven that, when $\vec{F}(\vec{x})$ is analytic, quadratic convergence to fixed points of Eq. (15) is obtained by the E-algorithm, without derivatives of $F$ and even when $F$ is not a contraction mapping. 
$p$ and r-algorithms. These two algorithms are related to the rational interpolation-extrapolation defined by $\mathrm{Eq}$. (5), but they can be derived directly from the Pade' approximants of type II. Let us write $S_{i}$ in the following manner:

$$
s_{i}=\frac{p_{0}+p_{1} x_{i}+p_{2} x_{i}^{2}+\ldots+p_{1} x_{i}^{1}}{q_{0}+q_{1} x_{i}+q_{2} x_{i}^{2}+\ldots+q_{m} x_{i}^{m}}
$$

where $x_{i}$ is often chosen equal to $i$ or $1 / i$ or some monotonic function of either of these. The extrapolation limit $(i \rightarrow \infty)$ is for $X_{i} \rightarrow 0$, in which case the ratio $p_{O} / q_{0}$ becomes the interesting quantity, or for $X_{i} \rightarrow a$, in which case we must choose $\ell=m$ to find $p_{\ell} / q_{\ell}$.

For the case $x_{i} \rightarrow \infty$ the ratio $p_{\hat{k}} / q_{g}$ can be calculated using the -algorithm $\left(\right.$ Wynn $\left.{ }^{3}\right)$ :

$$
\sigma_{-1}^{i}=0 ; \quad \rho_{0}^{i}=S_{i} ; \quad \rho_{k+1}^{i}=\rho_{k-1}^{j+1}+\frac{x_{i+k+1}-x_{i}}{\rho_{k}^{i+1}-\rho_{k}^{i}}
$$

The even index sequences $: \frac{i}{2 k}$ accelerate the calculation of the limit, whereas ihe odd index ones enter as an intermediate step into the recursive calculation (as in the --algorithm).

The case $x_{i} \rightarrow 0$ allows us to evaluate the $p_{0} / q_{0}$ ratio from diagonal $(:=m)$ and off-diagonai $(\Omega \neq m)$ approximants; the $r$-algorithm calculates il for a staircase sequence ${ }^{4}$ of type II approximants:

$$
\begin{gathered}
r_{-1}^{i}=0: \quad r_{0}^{i}=s_{i} ; \\
r_{k}^{i}=r_{k-1}^{i+1}+\frac{r_{i-1}^{i+1}-r_{k-1}^{i}}{\frac{x_{i}}{x_{i+k}}\left[1-\frac{r_{k-1}^{i+1}-r_{k-1}^{i}}{r_{k-1}^{i+1}-r_{k-2}^{i+1}}\right]-1}
\end{gathered}
$$

-algorithm and its generalizations. ${ }^{5}$ A further improvement to the determination of the limit of a sequence $\left(S_{i}\right)$ is achieved nowadays by applying the $\theta$-algorithm to $\varepsilon$ :

$$
\begin{gathered}
\theta_{-1}^{i}=0 ; \quad \theta_{o}^{i}=s_{i} ; \\
\theta_{2 k+1}^{i}=\theta_{2 k-1}^{i+1}+1 / \Delta \theta_{2 k}^{i} ; \\
\theta_{2 k+2}^{i}=\theta_{2 k}^{i+1}+\left(\Delta \theta_{2 k}^{i+1}\right)\left(\Delta \theta_{2 k+1}^{i+1} / \Delta^{2} \theta_{2 k+1}^{i}\right)
\end{gathered}
$$


where

$$
\Delta \mathrm{f}^{i}=\mathrm{f}^{\mathrm{i}+1}-\mathrm{f}^{\mathrm{i}} .
$$

As for the $\varepsilon$-algorithm, only the even numbered subscripts are expected to give approximations of $S$. In a very similar manner we deduce for the 0 :

$$
\begin{gathered}
\theta_{-1}^{i}=0 ; \quad \theta_{o}^{i}=s_{i} ; \\
\theta_{2 k+1}^{i}=\theta_{2 k-1}^{i+1}+D_{2 k+1}^{i} ; \\
\theta_{2 k+2}^{i}=\theta_{2 k}^{i+1}-D_{2 k+1}^{i} \frac{\Delta f_{2 k}^{i+1}}{\Delta D_{2 h+1}^{i}}
\end{gathered}
$$

where

$$
D_{2 k+1}^{i}=\frac{x_{i+2 k+2}-x_{i}}{\frac{i+1}{2 k+1}-\frac{i}{2 k+1}}
$$

The $\theta$ columns with even lower index give the convergent sequences. This new algorithm is a generalization of the $\theta$-algorithm derived from the $\varepsilon$ - and is one of the most powerful acceleration methods actually known. ${ }^{6}$ If we apply it to the F-algorithm we have:

$$
\begin{gathered}
\theta_{0}^{i}=s_{i} ; \\
\theta_{k+1}^{i}=\theta_{k-1}^{i+1}-\frac{\Delta \theta_{k-1}^{i+1}}{\Delta D_{k}^{i}} D_{k}^{i}
\end{gathered}
$$

with

$$
n_{k}^{i}=\frac{\Delta \theta_{k-1}^{i}}{\Delta z i-1, k} \cdot q_{k-1, k}^{i+1}
$$

and $g_{k, i}^{i}$ defined by Er. (7). 
IV. THE EFFECT OF THE BEAM-BEAM INTERACTION ON THE STABILITY AND POLAR TZATION OF A TEST PARTICLE (WEAK BEAM-STRONG BEAM) IN STORAGE RINGS

Bunched and unbunched beams undergo instantaneous periodic solicitations at their interaction points (intersection points for the case of intersecting rings) during accumulation in storage rings, hecause of the so-called beam-beam interaction. ${ }^{7}$

We analyze here a simplified version of this complicated problem: the betatron oscillations of a test particle belonging to a low density beam that interacts with a high delsity beam (weak-strong case). In a situation of this sort the beam-beam forces produce periodic instantaveous changes of the momentum components of the particie betatron motion and act on the spin precession around the static field, determining a sudden change of the spin components and consequently a variation of the polarization.

We introduce the betatron elongations $x(t), z(t)$ in the vertical plane perpendicular to the main revolution trajectory (assumed horizontal): both elongations are affected by the kick-effect in the case of head-on collisions (for instance in $e^{+}-e^{-}$and $p-\bar{p}$ rings), whereas only the vertical amplitude $z(t)$ feels the beam-beam force in the fixed angle (unbunched beams) collisions (ISR and ISABFLLF).

We must take into account other contributions: the synchrotron modulation of the tunes, the damping due to radiation, which is relevant mainly for electrons, and the synchrotron modulation of the beam-beam forces that is present in the bunched beams.

The betatron oscillations $x(t), z(t)$ can therefore be written as $\ddot{x}+2 \gamma_{x} \cdot \dot{x}+\omega_{x}^{2}\left[1-\lambda_{x} \phi_{x}(t)\right] x=-\hat{\xi}(t) \cdot x \cdot \phi(x \cdot x) \cdot \sum_{n}^{\infty} \delta(t-n \tau)(24)$ $\ddot{z}+2 \gamma_{z} \cdot \dot{z}+\omega_{z}^{2}\left[1-\lambda_{z} \phi_{z}(t)\right] z=-\hat{n}(t) \cdot z \cdot \psi(x \cdot z) \cdot \sum_{n}^{\infty} \delta(t-n \tau)(25)$ 
where we assumed that the first collision between the beams takes place at the instant $t=\tau=\mathrm{T} / \mathrm{m}$, with $\mathrm{T}=2 \pi / \omega_{R}$ being the revolution and $\mathrm{m}$ the number of interactions (intersections) per revolution. Here, $\phi_{x}(t)$, $\phi_{z}(t)$ are periodic functions with period $T_{S}=2 \pi / \omega_{s}$ equal to the synchrotion oscillation period and they satisfy the requirement that $\left|\phi_{x}\right|,\left|\phi_{z}\right| \lesssim 1$ with $\left|\lambda_{x}\right|,\left|\lambda_{z}\right| \ll 1$. Also, $\hat{\xi}(t), \hat{n}(t)$ are the synchrotron modulated beam-beam couplings: they are periodic with a frequency which is twice the synchrotron frequency. ${ }^{8}$ A convenient choice is $\phi_{X}(t)=\phi_{z}(t)=\cos \omega_{s} t ; \quad \xi(t)=\xi_{0}\left(1+\Lambda_{x} \cos 2 \omega_{s} t\right) ; \hat{\eta}(t)=$ $-n_{0}\left(1+\Lambda_{z} \cos 2 \omega_{s} t\right)$ with $\Lambda_{x}, \Lambda_{z} \cong 1$.

The functions $\phi(x, z), \psi(x, z)$ are related to the electric and magnetic fields acting on the test particle at the instant of the kick? and can be derived easily in an analytical form once.the charge and current densities of the beam are assigned. If thece exhibit, as usually is assumed on physical grounds, a Gaussian distribution in $x$ and $z$, and are constant along the s coordinate, the functions $\phi$ and $\psi$ achieve a simple integral representation ( $a, b$ being rms Gaussian parameters of the charge density):

$$
\begin{aligned}
& \phi(x, z)=\int_{0}^{\infty} d t\left[a^{2}+t\right]^{-1 / 2} \cdot\left[b^{2}+t\right]^{-3 / 2} \exp \left\{-\frac{x^{2}}{a^{2}+t}-\frac{z^{2}}{b^{2}+t}\right\}, \\
& \psi(x, z)=\int_{0}^{\infty} d t\left[a^{2}+t\right]^{-3 / 2} \cdot\left[b^{2}+t\right]^{-1 / 2} \exp \left\{-\frac{x^{2}}{a^{2}+t}-\frac{z^{2}}{b^{2}+t}\right\} .
\end{aligned}
$$

The coupling constants $\xi_{0}$, $\eta_{0}$ depend on the particle density (on the number of particles per bunch, $N_{B}$, in the bunched beam case), the type of particles, and their average scalar velocity along the main trajectory; for electrons and positrons we have

$$
\xi_{0}=\eta_{0}=\frac{2 N_{B}{ }^{r} e}{\gamma B^{2}}
$$

where $r_{e}$ is the classical electron radius.

If we are given two linearly independent solutions of the homogeneous equations, obtained from Eqs. (24) and (25) by dropping the beam-beam forces (which means $\hat{\xi} \equiv \hat{\eta}=0$ ), then we can solve the system (24), (25) exactly. Indeed, after changing variable from to $\theta=\omega_{k} t$ and rewriting the differential system (26), (27) with the new derivatives $\left(x^{\prime}=\frac{d x}{d \theta}, x^{\prime \prime}=\frac{d^{2} x}{d \theta^{2}}\right.$, etc.) we find the following equations:

$$
\begin{aligned}
& x^{\prime \prime}+2 \Gamma_{x^{\prime}}+\omega_{x}^{2}\left[1-\lambda_{x} \phi_{x}\left(\theta / \omega_{R}\right)\right] x=-x \cdot \phi \cdot \sum_{n}^{\infty} \xi_{n} \delta\left(\theta-n \theta_{m}\right),(22) \\
& z^{\prime \prime}+2 \Gamma_{z^{\prime}} z^{\prime}+\omega_{z}^{2}\left[1-\lambda_{z} \phi_{z}\left(\theta / \omega_{R}\right)\right] y=-z \cdot \psi \cdot \sum_{n}^{\infty} n_{n} \delta\left(\theta-n \theta_{m}\right)(30)
\end{aligned}
$$


where $\theta_{m}=\frac{2 \pi}{m}=\tau \omega_{R}, \xi_{n}=\xi_{0}\left[1+\Lambda_{x} \cos \left(\frac{2 \omega_{s}}{\omega_{R}} \theta_{m} n\right)\right]$, and $n_{n}=n_{0}\left[1+\Lambda_{z} \cos \left(\frac{2 \omega_{s}}{w_{R}} \theta_{m} n\right)\right]$.

At this point we introduce the sequences of functions $(k=1,2, \ldots n) x_{k}(\theta)=A_{k} M(\theta)+B_{k} N(\theta), z_{k}(\theta)=C_{k} P(\theta)+D_{k} Q(\theta)$, with $M, N$ and $P, Q$ being linearly independent solutions of the homogenous system associated with Eqs. (29), (30); and we make the physical solution of Eas. (29) and (30), $\mathrm{x}(\theta), \mathrm{z}(\theta)$, coincide with $x_{k}, y_{k}$ in the open interval $\left[\frac{2 \pi}{m}(k-1), \frac{2 \pi}{m} k\right]$.

Integration of the system (29), (30) along the nth infinitesimal interval $\left[\frac{2 \pi}{m} n-\varepsilon, \frac{2 \pi}{m} n+\varepsilon\right]$, joined with the continuity conditions for the elongations $x(\theta), z(\theta)$, gives us the following formulae:

$$
\begin{gathered}
x_{n+1}\left(\frac{2 \pi}{m} n\right)=x_{n}\left(\frac{2 \pi}{m} n\right), \\
z_{n+1}\left(\frac{2 \pi}{m} n\right)=z_{n}\left(\frac{2 \pi}{m} n\right), \\
x_{n+1}^{\prime}\left(\frac{2 \pi}{m} n\right)=x_{n}^{\prime}\left(\frac{2 \pi}{m} n\right)-\xi_{n} x_{n}\left(\frac{2 \pi}{m} n\right) \phi\left[x_{n}\left(\frac{2 \pi}{m} n\right), z_{n}\left(\frac{2 \pi}{m} n\right)\right], \\
z_{n+1}^{\prime}\left(\frac{2 \pi}{m} n\right)=z_{n}^{\prime}\left(\frac{2 \pi}{m} n\right)-\eta_{n} z_{n}\left(\frac{2 \pi}{m} n\right) \psi\left[x_{n}\left(\frac{2 \pi}{m} n\right), z_{n}\left(\frac{2 \pi}{m} n\right)\right] .
\end{gathered}
$$

[Obviously $x_{n+1}^{\prime}\left(\frac{2 \pi}{\pi !} n\right) \equiv x^{\prime}\left(\frac{2 \pi}{m} n+\varepsilon\right) \equiv x^{\prime(+)}\left(\frac{2 \pi}{m} n\right)$ and $\left.x_{n}^{\prime}\left(\frac{2 \pi}{m} n\right) \equiv x^{\prime}\left(\frac{2 \pi}{m} n-\varepsilon\right) \equiv x^{\prime}-\right)\left(\frac{2 \pi}{m} n\right)$, and similarly for $\left.z^{\prime} \cdot\right]$

Eqs. (31) through (34) give us the constants $A_{n+1}, B_{n+1}, C_{n+1}$, $D_{n+1}$ as linear recursive functions of $A_{n}, B_{n}, C_{n}, D_{n}^{n+1}$ ur equivalently the elongations $x\left(\frac{2 \pi n}{m}\right), z\left(\frac{2 \pi n}{m}\right)$ and their right-handed derivatives

$x^{\prime(+)}\left(\frac{2 \pi}{m} n\right): z^{(+)}\left(\frac{2 \pi}{m} n\right)$ in terms of $x\left[\frac{2 \pi}{m}(n-1)\right], z\left[\frac{2 \pi}{m}(n-1)\right]$, $x^{\prime(+)}\left[\frac{2 \pi}{m}(n-1)\right], z^{\prime(+)}\left[\frac{2 \pi}{m}(n-1)\right]:$ 


$$
\begin{aligned}
& x\left(\frac{2 \pi}{m} n\right)=\frac{N_{0}^{1} M\left(\frac{2 \pi}{m}\right)-M_{0}^{1} N\left(\frac{2 \pi}{m}\right)}{M_{0} N_{0}^{\prime}-M_{0}^{\prime} N_{0}} \times\left[\frac{2 \pi}{m}(n-1)\right] \\
& +\frac{M_{o} N\left(\frac{2 \pi}{m}\right)-N_{0} M\left(\frac{2 \pi}{m}\right)}{M_{0} N_{0}^{\prime}-M_{0} N_{0}} \times{ }^{(+)}\left[\frac{2 \pi}{m}(n-1)\right], \\
& x^{\prime}(+)\left(\frac{2 \pi}{m} n\right)=\frac{N_{0}^{\prime} M^{\prime}\left(\frac{2 \pi}{m}\right)-M_{0}^{\prime} N^{\prime}\left(\frac{2 \pi}{m}\right)}{M_{0} N_{0}^{\prime}-M_{0}^{\prime} N_{0}} \times\left[\frac{2 \pi}{m}(n-1)\right] \\
& +\frac{-N_{0} M^{\prime}\left(\frac{2 \pi}{m}\right)+M_{0} N^{\prime}\left(\frac{2 \pi}{m}\right)}{M_{0} N_{0}^{\prime}-M_{0}^{\prime} N_{0}} \times{ }^{\prime(+)}\left[\frac{2 \pi}{m}(n-1)\right] \\
& -\xi_{n} x\left(\frac{2 \pi}{m} n\right) \phi\left[x\left(\frac{2 \pi}{m} n\right), z\left(\frac{2 \pi}{m} n\right)\right]
\end{aligned}
$$

where $M_{0}=M(0), M_{0}^{\prime}=M^{\prime}(0), N_{0}=N(0), N_{0}^{\prime}=N^{\prime}(0)$, and similarly for $z\left(\frac{2 \pi}{m} n\right), z^{\prime(+)}\left(\frac{2 \pi}{m} n\right)$ with the substitutions $M+P, N+0, n+\eta$, and $\phi \rightarrow \psi$.

Note that the coupling between the two oscillators $x, z$ is due to the nonlinear beam-beam terms $\phi$ and $\psi$. It is at this point that the algorithms illustrated in Sections II and III can be applied to search for the temporal asymptotic behavior of the moduli of the elongations $x(\theta), y(\theta)$, or equivalently in the discrete variable $n$, for $n \rightarrow \infty$ in the various different physical cases. To be more explicit, once we are giver, the ring parameters and the input data (tune, frequency of revolution, initial kinematical conditions of the test particle, tuneshift, etc.), Eas. (35), (36) and the analogous ones for $7, z^{(+)}$ give us the value of the elongation $x$ after the nth kick in terms of the values of $x, z, x^{\prime}, z^{\prime}$ after the preceding one. One can therefore consider the moduli of the maximal elongations in each interval $\left[\frac{2 \pi n}{m}, 2 \pi(n+1)\right]$ and take the limit of the sequence so obtained for $n \rightarrow \infty$. The limit, if it exists, gives us an answer on the stability of the betatron oscillations. The amount of information that our simulation model produces through the detailed numerical computation of the values $x(2 \pi n / m), z(2 \pi n / m)$ with $n$ going from 1 to $10^{6}$ appears to be sufficient for a practical evaluation of the limit. 9

The number of revolutions in the ring for which stability against beam-beam interaction is requested is of the order of $10^{10}, 10^{11} ;$ and a numerical computation of the formulae (35), (36), etc., from $n=1$ to $\mathrm{n}=10^{10}, 10^{11}$ seems impractical and nonrealistic on a digital computer at present. Instead, use of the proposed method, once the first $10^{6}$, $1 n^{7}$ values of the amplitudes have been memorized, seems able to supply the answer sought, namely whether or not stability is obtained for a 
given set of input data. Simple models that may already simulate the behavior of cxisting and future storage rings can be obtained from the system described by Eqs. (24), (25). In particular, one can derive the one-dimensional case valid for fixed angle collisions between protons (ISR and ISABELLE) characterized by beam-beam interactions act ing only on the vertical oscillator. In this situation, we neglect damping and keep or do not keep the synchrotron modulation of the tune and of the tuneshift according to the two situations of bunched and unbunched beams.

A very good approximation to the actual beam-beam force ${ }^{7}$ in these two cases is given by a simplified expression of the $\psi(x, z)$ function:

the error function $\phi(z)=\frac{2}{\sqrt{\pi}} \int_{0}^{z / \sqrt{2} \cdot 0} \mathrm{dt} \exp \left\{-t^{2}\right\}$ or $\tilde{\phi}(z)=\frac{1-e^{-z^{2} / 2 \sigma^{2}}}{z \sigma^{2}}$ for the unbunched and the bunched one-dimensional models respectively. Analytical details are given in the Appendix. Note, however, that the approach based on extrapolation to the limit $c$ an handle the one-dimensional and the two-dimensional cases in a similar manner. But the physical results and conclusions could be very different in the two cases, because the coupling among different degrees of freedom may generate eftects that are absent in the onedimensional case.

Effects on Polarized Beams. Let us consider the test particle of a low-density beam in a certain spin polarization state. In electronpositron storage rings the beam becomes polarized spontaneously because of radiation emission (magnetic dipole transition), reaching a very high degree of polarization, along this static bending field $\vec{b}_{Q}$, as various authors have pointed out; in proton, antiproton or heavier particle rings the polarization of the beam must be obtained by different ad-hoc methods. ${ }^{10}$ If there is a degree of polarization $P_{0}$ under regime conditions without the beam-beam forces, how is it modified by their presence?

\footnotetext{
* As far as the numerical calculation is concerned, one picks up a subset of input memorized data with particular "a priori" established criteria and gives them to the various algorithms as an input, thus producine several outputs which must be compared with one another. Arnold's diffusion is rigorously absent when the perturbed betatron oscillation is orle-dimensional, and is normally expected to be very weak for cases with higher degrees of freedom.
} 
We develop here a semiclassical formulation of the motion of the spin, first introduced by Thomas and later developed within the relativistic quantum-mechanical scheme by other authors, 11 our final objective being to determine the spin-behavior after many revolutions (order of $10^{11}$ ). The operator $\vec{S}$, representing the spin operator in the rest-frame of the particle and there associated with the intrinsic magnet ic moment $\vec{\mu}_{0}=$ geh $\mathbb{S} / 2 \mathrm{mc}$, in the lab system undergoes the following equation of motion:

$$
\frac{d \vec{S}}{d t}=\frac{e}{m c} \vec{S} \times\left[\left(a+\frac{1}{\gamma}\right) \vec{B}-\frac{a \gamma}{\gamma+T} \vec{B}(\vec{B} \cdot \vec{B})-\left(a+\frac{1}{a+T}\right) \vec{B} \times \vec{E}\right]
$$

where $a$ is the anomaly: $a=(g-2) / 2$.

We separate the static field ${ }_{0}$ in the lab system from the fields created by the dense beam in periodic collisions with the test particle; under the physical assumption that the collision time is very small in comparison with the precession of the spin around $\hbar_{0}$, we can write for $\vec{B}$ and $\frac{\hat{E}}{\mathrm{H}}$ the following formulae:

$$
\begin{gathered}
\vec{B}=\vec{B}_{0}+\vec{B}_{1} \cdot \sum_{-\infty}^{\infty} \delta(t-n \tau), \\
\vec{E}=\vec{E}_{1} \cdot \sum_{n}^{\infty} \delta(t-n \tau) .
\end{gathered}
$$

$B_{0}$ is static and should be uniform in an ideal ring but, according to our reasoning, could also be taken as space dependent; $\mathrm{B}_{1}$ and $\mathrm{E}_{1}$ have space-functional dependence, already considered in the betatron motion, related to the charge and current distributions in the dense beam.

We may change variables, as before, $\theta=\omega_{\mathrm{R}} \mathrm{t}$, and introduce the infinite sequence $\left\{\vec{s}_{k}(\theta)\right\}(k=1,2, \ldots)$ defined as follows: the vector $s_{k}(\theta)$ coincides with $\xi(\theta)$ in the spin interval $\frac{2 \pi}{m}(k-1)<\theta<$ $\frac{2 \pi}{m} \mathrm{k}$, and therefore verifies the equation

$$
\frac{d s_{k}}{d \theta}=\frac{e \omega_{R}}{m c} \vec{s}_{k} \times\left[\left(a+\frac{1}{\gamma}\right) \vec{B}_{o}-\frac{a \gamma}{\gamma+T} \vec{B}\left(\vec{B} \cdot \vec{B}_{o}\right)\right]
$$

and is uniquely assigned in terms of its value at $\theta=\frac{2 \pi}{m}(k-1)$; more precisely

$$
s_{k}(\theta)=t(\theta), s_{k}\left[\frac{2 \pi}{m}(k-1) j\right.
$$

where $\xi_{k}\left[\frac{2 \pi}{m}(k-1)\right]=\vec{s}\left[\frac{2 \pi}{m}(k-1)+\varepsilon\right], \quad \varepsilon+0$.

If $B_{0}$ is uniform, perpendicular to the orbit $\left(B \cdot B_{0}\right) \equiv 0$, the solution $(41)$ is a simple precession of angular velocity $\hat{\omega}=\frac{e}{\mathrm{mc}}\left[a+\frac{1}{\gamma}\right] \hat{B}_{o}=\omega_{\tilde{z}}(\hat{z}=$ unit vector of the $z$-vertical axis $):$ 


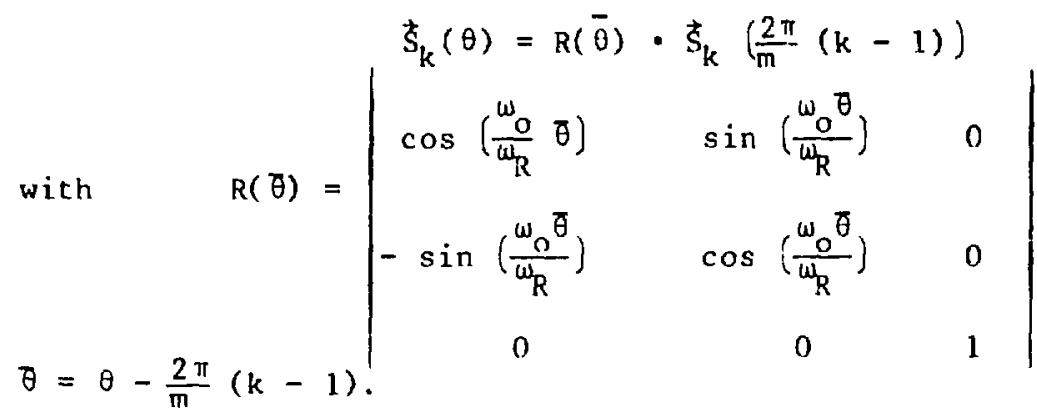

We can now integrate Eq. (37) along an infinitesimal time interval $(n \tau-\varepsilon, n \tau+\varepsilon$ ) or equivalent ly, using the $\theta$ variable, along the interval $\left[\frac{2 \pi}{m} n-\varepsilon, \frac{2 \pi}{m} n+\varepsilon\right]$ :

$$
\vec{s}\left[\frac{2 \pi}{m} n+\varepsilon\right]-\vec{s}\left[\frac{2 \pi}{m} n-\varepsilon\right]=\vec{S}\left(\frac{2 \pi}{m} n\right) \times 2 \vec{W}_{n}
$$

where $\vec{\xi}\left(\frac{2 \pi}{m} n\right)$ means $\frac{1}{2}\left[\vec{s}\left(\frac{2 \pi}{m} n+\varepsilon\right)+\vec{s}\left(\frac{2 \pi}{m} n-\varepsilon\right)\right]$, and the vector $\vec{h}_{n}$ is

$$
\begin{gathered}
\vec{W}_{n}=\left(a+\frac{1}{Y}\right) \vec{B}_{1}\left[\vec{x}\left(\frac{2 \pi}{m} n\right)\right] \\
-\frac{a Y}{Y+T} \vec{B}_{n} \cdot\left\{\vec{B}_{n} \cdot \vec{B}_{1}\left[\vec{x}\left(\frac{2 \pi}{m} n\right)\right]-\left(a+\frac{1}{Y+T}\right) \vec{B}_{n} \times \vec{E}_{1}\left[x\left(\frac{2 \pi}{m} n\right)\right]\right\} .
\end{gathered}
$$

B can be made to coincide with the velority of the main orbit motion and is known. $\vec{x}\left[\frac{2 \pi}{m} \mathrm{n}\right]$ is the position vector of the particles in the lab system and can be considered rigorously the superposition of the revolution $\vec{x}_{R}(t)$ with the betatron-synchrotron oscillations;

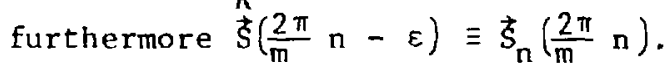

From Eq. (43) and our definition of $\$\left(\frac{2 \pi}{m} n\right)$ we obtain the representation of the spin operator after the nth kick:

$$
\Xi\left(\frac{2 \pi}{m} n+\varepsilon\right)=\left[1+\vec{W}_{n} x\right]^{-1}\left[1-\vec{W}_{n} x\right] \cdot \xi\left(\frac{2 \pi}{m} n-\varepsilon\right)
$$

and finally the relation between the $(n-1)$ and the nth kick:

$$
\vec{S}\left(\frac{2 \pi}{m} n+\varepsilon\right)=\left[1+\vec{k}_{n} x\right]^{-1}\left[1-\vec{k}_{n} x\right] \cdot \vec{F}\left(\frac{2 \pi n}{m}, \vec{S}\left[\frac{2 \pi}{m}(n-1)+\varepsilon\right]\right) \cdot(46)
$$


The case of homogeneous $\vec{B}_{0}$ gives

$$
\xi\left(\frac{2 \pi}{m} n+\varepsilon\right)=\left[1+\vec{k}_{n} \times\right]^{-1}\left[1-\vec{W}_{n} x\right] \cdot \xi\left(\frac{2 \pi}{m}\right) \cdot \xi\left[\frac{2 \pi}{m}(n-1)+\varepsilon\right] \cdot(47)
$$

Note that the vector $\vec{W}_{n}$ contains the whole nonlinear contribution of the beam-beam interaction and couples the spin operator with the orbital classical motion.

The operator $\vec{s}$ is considered an Heisenberg operator, the evaluation of the matrix elements $\langle f|\vec{s}(t)| i\rangle$ where $|f\rangle$ and $|i\rangle$ are time-independent spin states is straight forward and because of the linearity of the spin operator Eq. (37), gives the following represent ation:

$\left.\langle f|\vec{S}(t)| i\rangle\right|_{\theta=\frac{2 \pi n}{m}+\varepsilon}=\left[1+\vec{W}_{n} x\right]^{-1}\left[1-\vec{W}_{n} x\right] \cdot \vec{F}\left(\frac{2 \pi n}{m}\right),\left.\langle f|\vec{S}| i\rangle\right|_{\theta=\frac{2 \pi(n-1)}{m}+}$ and similarly [Eo. (47)] for 声 uniform field.

Knowledge of the matrix elements $\langle\mathfrak{f}|\mathrm{J}| \mathrm{i}\rangle$ for any $\theta$ provides all the information on the states of polarization of the test particle at the corresponding time $t=\theta / \mathrm{u}_{R}$. If we are given the polarization at $t=0$ we can calculate it at all instants $t$, and in particular in the nth interval $\frac{2 \pi}{m}(n-1)<\theta<\frac{2 \pi}{m} n$. The extrapolation algorithms in principle allow, at this point, calculation of the efrects of the beambeam interaction on the polarized test particle under regime conditions $(n+\infty)$. 


\section{REFERENCES}

1. C. Breainski, Numer. Math. 35, 175-87 (1980).

2. D. Shanks, Numer. Math. Phys. 34, 1-42 (1955).

3. P. Wynn, Proc. Cambridge Philos. Soc. 52, 66371 (1956); G. Baker Jr., Essentials on Padé Approximants, Academic Press, New York, 1975.

4. J. Stoer, Numer. Math, 3, 285-304 (1961).

5. C. Brezinski, C.R. Acad. Sci., Paris 273A, 727-730 (1971); see also ref. $i$, p. 186 .

6. D.A. Smith, SIAM J. Numer. Ana1. 6, 223-240 (1970).

7. J.C. Herrera, in Electromagnetic Interactions in Colliding Beams in Storage Rings, M. Month and J.C. Herrera, Editors, American Institute of Physics, New Yort. 1979.

8. J. Tennyson, p. 189 in ref. 7 .

9. A. Pascolini and M. Pusterla, IffeE Trans. Nucl. Sci. N.S. 28, p.2543 (1981); A. Pascolini, M. Pusterla, and L. Stanes, Lettere Nuovo Cimento $36,6,217$ (1981).

10. A.A. Sokolov and I.M. Ternov, Dokl. Akad. Nauk SSSR 153, 1052 (1963) [(Sov. Phys. - Dokl. 8, 1203 (1964)]; V.N. Baier, C. Usp. Fiz, Nauk. 105, 441 (1971) TSov. Phys. - Usp. 14, 695 (1972)]; J.D. Jackson, Rev. Mor. Phys. 48, (3), 417 (1976); A.W. Chao, Nucl. Instrum. Methods $180,2 \overline{9}$, (1981); E.D. Courant and R.D. Ruth, BNL 51270, Sept. $1 \overline{980}$.

11. Thomas, L.T.; Phil. Mag. 3, 1 (1927); V. Bargmann, L. Michel, and V.L. Telegdi, Phys. Rev. Lett. 2, 435 (1959).

12. M. Abramowitz and I.A. Stegun, Handbook of Mathematical Functions, pp. 727-730, Dover, New York, $1 \overline{970}$. 
Choices of the $g i(n)$ that reproduce well-known $t$ ransformations

$$
\begin{aligned}
& g_{i}(n)=X_{n}^{I} \text { Richardson extrap. process ( } X_{n} \text { being a given } \\
& \text { auxiliary sequence of parameters) } \\
& g_{i}(n)=x_{n+i-1} \quad \text { r-transf. } \\
& g_{i}(n)=\left(x_{i}\right)^{n} \quad \text { Surmation process } \\
& g_{i}(n)=\Delta s_{n+i-1}\{\quad \text { Shanks transf. } \\
& g_{i}(n)=\Delta^{i} S_{n} \\
& g_{i}(n)=\left(\Delta s_{n}\right) i \quad \text { Germain-Bome transf. } \\
& g_{i}(n)=\left[x_{n}^{i-1} / g(n)\right] \Delta S_{n} \text { Levin generalized transf. ( } g \text { being given) } \\
& \left.\begin{array}{l}
g_{i}(n)=x_{n} \\
g_{i}(n)=\Delta s_{n+i-2}
\end{array}\right\} \quad \text { so-called process } p
\end{aligned}
$$

Particular Cases of the Betatron Motion Equations

If we neglect the damping term (proton case) and assume $\phi(t)=\cos \omega_{s} t, \quad \hat{\xi}=\xi_{o}\left(1+\cos 2 \omega_{s} t\right)$ dealing with the one-dimensional bunched problem, we have the equation of the vertical elongation $z(t)$ only, which in the variable $\theta=\omega_{R} t$ becomes

$$
z^{\prime \prime}+v_{0}^{2}\left(1-\lambda \cos \frac{\omega_{s}}{\omega_{k}^{\omega}} \theta\right) z=-z \phi(z) \cdot \sum_{n}^{\infty} \xi_{n} \delta\left(\theta-\theta_{m} \cdot n\right)
$$

with $\phi(z)=\frac{1-e^{-z^{2} / 2 \sigma^{2}}}{z^{2} / 2 \sigma^{2}}$.

Equation (2a) has an exact solution in terms of the Mathieu functions. ${ }^{2}$ More specifically we may write $z(\theta)$ as

$$
z(\theta)=A M(\theta)+B N(\theta)
$$

where $M(\theta)$ and $N(\theta)$ are the real and the imaginary parts of the Mathieu function $F$, and $A$ and $B$ are real arbitrary constants. The relations between the elongation $z(2 \pi n / m)$, its derivative $z '[(2 \pi n / m)+\varepsilon]$, and $z[(2 \pi / m)(n-1)]$ and $z^{\prime}[(2 \pi / m)(n-1)+\varepsilon]$ are: 


$$
\begin{gathered}
z\left(\frac{2 \pi}{m} n\right)=\alpha z\left[\frac{2 \pi}{m}(n-1)\right]+\beta_{z}^{\prime}\left[\frac{2 \pi}{m}(n-1)+\varepsilon\right] \\
z^{\prime}\left[\frac{2 \pi}{m} n+\varepsilon\right]=\bar{\alpha}_{z}\left[\frac{2 \pi}{m}(n-1)\right]+\beta_{z}^{\prime}\left[\frac{2 \pi}{m}(n-1)+\varepsilon\right. \\
\quad-\xi_{n} z\left(\frac{2 \pi n}{m}\right) \cdot \phi\left[z\left(\frac{2 \pi n}{m}\right)\right] \\
\alpha=\left[N_{0}^{\prime} M\left(\frac{2 \pi}{m}\right)-M_{0}^{\prime} N\left(\frac{2 \pi}{m}\right)\right]\left[M_{0} N_{0}^{\prime}-M_{0}^{\prime} N_{0}\right]^{-1} \\
\beta=\left[M_{0} N\left(\frac{2 \pi}{m}\right)-N_{0} M\left(\frac{2 \pi}{m}\right)\right]\left[M_{0} N_{0}^{\prime}-M_{0}^{\prime} N_{0}\right]^{-1} \\
\bar{\alpha}=\left[N_{0} M^{\prime}\left(\frac{2 \pi}{m}\right)-M_{0}^{\prime} N^{\prime}\left(\frac{2 \pi}{m}\right)\right]\left[M_{0} N_{0}^{\prime}-M_{0}^{\prime} N_{0}\right]^{-1} \\
\bar{B}=\left[-N_{0} M^{\prime}\left(\frac{2 \pi}{m}\right)+M_{0} N^{\prime}\left(\frac{2 \pi}{m}\right)\right]\left[M_{0} N_{0}^{\prime}-M_{0}^{\prime} N_{0}\right]^{-1}
\end{gathered}
$$

where $N_{0}=N(0), M_{0}=M(0), N_{0}^{\prime}=N^{\prime}(0)$, and $M^{\prime}(0)=M_{0}^{\prime}$. Because $\lambda \ll 1$ $M(\theta)=\cos \left(\bar{\nu} \frac{\omega_{S}}{\omega_{R}} \theta\right)-\frac{q}{4(\bar{\nu}+1)} \cos \left[\theta \frac{\omega_{S}}{\omega}\left(\frac{\bar{\nu}}{2}+1\right)\right] \frac{q}{4(\bar{v}-1)} \cos \left[\theta \frac{\omega_{S}}{\omega_{R}}\left(\frac{\bar{\nu}}{2}-1\right)\right]$ $N(\theta)=-\sin \left(\bar{v} \frac{\omega_{S}}{\omega_{R}} \theta\right)+\frac{q}{4(\bar{v}+1)} \sin \left[\theta \frac{\omega_{S}}{\omega_{R}}(\bar{v}+1)\right]$

$$
-\frac{q}{4(\bar{v}-1)} \sin \left[\frac{\omega_{S} \theta}{\omega_{R}}\left(\frac{\bar{v}}{2}-1\right)\right] \text {, }
$$

and, at $\theta=0$,

$M_{0}=1+\frac{q}{2\left(\bar{v}^{2}-1\right)} ; \quad N_{0}=0 ; \quad M_{0}^{\prime}=0 ; N_{0}^{\prime}=\frac{\omega_{S}}{\omega_{R}}\left[-\bar{v}+q \frac{\bar{v}}{2\left(\bar{v}^{2}-1\right)}\right]$

where $q=\lambda / 2 \bar{v}^{2}$ and $\bar{v}^{2}=v_{\mathrm{o}}^{2}\left(4 \omega_{\mathrm{R}}^{2} / \omega_{\mathrm{S}}^{2}\right)$.

The linit $\lambda+0$ gives the simple harmonic betatron oscillation plus the nonlinear effect of the beam-heam forces. This siniplified situation is physically valid for the unbunched beams, as a good approximation. However, one must substitute $\phi(z)$ in Eq. (2a) with the error function $\frac{2}{\sqrt{2}} \int_{0}^{z / \sqrt{2} \cdot \sigma} d t \exp \left(-t^{2}\right)$. 